\title{
On a nonlocal moving frame approximation of traveling waves
}

\author{
Jose M. Arrieta ${ }^{\mathrm{a}}$ Maria López-Fernández ${ }^{\mathrm{b}}$ Enrique Zuazua ${ }^{\mathrm{c}, \mathrm{d}}$ \\ a Departamento de Matemática Aplicada. Universidad Complutense de Madrid, 28040 Madrid, Spain. \\ ${ }^{\mathrm{b}}$ Institut für Mathematik. Universität Zürich. Winterthurerst. 190, CH-8057 Zurich, Switzertland \\ ${ }^{\mathrm{c}}$ Ikerbasque, Basque Foundation for Science, Alameda Urquijo 36-5, Plaza Bizkaia, 48011, Bilbao, Basque Country, Spain \\ ${ }^{\mathrm{d}}$ BCAM - Basque Center for Applied Mathematics, Bizkaia Technology Park 500, 48160, Derio, Basque Country, Spain
}

\begin{abstract}
The profiles of traveling wave solutions of a 1-d reaction-diffusion parabolic equation are transformed into equilibria of a nonlocal equation, by means of an appropriate nonlocal change of variables. In this new formulation both the profile and the propagation speed of the traveling waves emerge as asymptotic limits of solutions of a nonlocal reaction-diffusion problem when time goes to infinity. In this Note we make these results rigorous analyzing the well-posedness and the stability properties of the corresponding nonlocal Cauchy problem. We also analyze its truncation to a finite interval with consistent boundary conditions. For large enough intervals we show that there is an asymptotically stable equilibrium which approximates the profile of the traveling wave in $\mathbb{R}$. This leads to efficient numerical algorithms for computing the traveling wave profile and velocity of propagation.
\end{abstract}

\section{Résumé}

Dans cette Note nous considérons le développement de méthodes permettant de préciser aussi bien les profils que la vitesse des ondes progressives pour des équations de reaction-diffusion, modélisées par des équations paraboliques semi-linéaires à une dimension d'espace. Moyennant un changement de variable non-local, les profils deviennent des solutions stationnaires d'un problème d'évolution non-local. Nous démontrons que, dans cette nouvelle formulaison, aussi bien les profils que les vitesses de propagation des ondes progressives deviennent des états stationnaires asymptotiques stables lorsque le temps tend vers l'infini. On analyse aussi la troncature de ce nouveau problème non-local de Cauchy en espace, à un intervalle d'espace fini. Lorsque l'intervalle d'espace tronqué est assez grand on montre qu'il existe un état stationnaire unique et que si l'intervalle tend vers la droite réelle entière, l'état stationnaire converge vers le profil de l'onde progressive. Ceci permet de développer des méthodes numériques efficaces de calcul des profils et vitesses de ces ondes progressives nonlinéaires.

\section{Version française abrégée}

On considère l'équation de réaction-diffusion :

$$
\left\{\begin{array}{l}
u_{t}(x, t)=u_{x x}(x, t)+f(u(x, t)), \quad-\infty<x<+\infty, \quad t>0, \\
u(x, 0)=u_{0}(x) .
\end{array}\right.
$$

Sous des hypothèses convenables sur la nonlinéarité $f$ on sait qu'il existe une unique solution de la forme onde progressive $u(x, t)=\Phi(x-c t)$ (voir [8]). Le profil $\Phi$ et la vitesse de propagation $c$ vérifient

$$
\left\{\begin{array}{l}
\Phi^{\prime \prime}(\xi)+c \Phi^{\prime}(\xi)+f(\Phi(\xi))=0, \quad-\infty<\xi<+\infty, \\
0 \leq \Phi \leq 1, \quad \Phi(-\infty)=0, \Phi(+\infty)=1, \quad \Phi^{\prime}>0
\end{array}\right.
$$

Email addresses: arrieta@mat.ucm.es (Jose M. Arrieta), maria.lopez@math.uzh.ch (Maria López-Fernández), zuazua@bcamath.org (Enrique Zuazua). 
et sont couplés par l'équation

$$
c=-\frac{F(1)}{\left\|\Phi^{\prime}\right\|_{L^{2}(\mathbb{R})}^{2}}
$$

avec $F(u)=\int_{0}^{u} f(s) d s$.

On considère alors le problème non-local

$$
v_{t}=v_{x x}-\frac{F(1)}{\left\|v_{x}\right\|_{L^{2}(\mathbb{R})}^{2}} v_{x}+f(v), \quad x \in \mathbb{R}, t>0 ; \quad v(x, 0)=u_{0}(x) .
$$

Naturellement, aussi bien les profils que les vitesses de propagation des ondes progressives deviennent des états stationnaires de ce nouveau problème. Le premier résultat de cette Note assure que cet état est stable et donc limite asymptotique lorsque le temps tend vers l'infini pour des données initiales souhaitables.

On analyse ensuite la troncature de ce nouveau problème non-local de Cauchy en espace à un intervalle d'espace fini $(-L, L)$, avec des conditions aux limites de Dirichlet de la forme $v(-L)=0, v(L)=1$. Lorsque l'intervalle d'espace tronqué est assez grand, on démontre qu'il existe un état stationnaire unique qui est exponentielement stable. On montre aussi que, lorsque l'intervalle tend vers la droite réelle t entière, cet etat stationnaire converge vers le profil de l'onde progressive.

Ceci permet de développer des méthodes numériques efficaces de calcul des profils et vitesses de ces ondes nonlinéaires. Il suffit, en effet, de résoudre l'équation tronquée non-local en temps pour récuperer le profil et la vitesse de l'onde progressive. On exhibe l'efficacité de cette méthode par diverses simulations numériques.

\section{Introduction}

We address the problem of the effective computation of traveling wave solutions emerging from parabolic semilinear equations on the real line. We consider the prototype reaction-diffusion equation:

$$
\left\{\begin{array}{l}
u_{t}(x, t)=u_{x x}(x, t)+f(u(x, t)), \quad-\infty<x<+\infty, \quad t>0, \\
u(x, 0)=u_{0}(x),
\end{array}\right.
$$

where the nonlinearity $f$ is of bistable type as in [8], that is, $f \in C^{1}(\mathbb{R})$ with $f(0)=f(1)=0, f^{\prime}(0)<0, f^{\prime}(1)<0$ and there exists $\alpha \in(0,1)$ such that $f(u)<0$ for $u \in(0, \alpha)$ and $f(u)>0$, for $u \in(\alpha, 1)$.

A traveling wave is a solution of (4) of the type $u(x, t)=\Phi(x-c t)$ where the function $\Phi$ is the profile of the traveling wave and $c$ is its speed of propagation. We will be mainly interested in "monotone" traveling waves (monotonic $\Phi$ ) connecting the two equilibria 0 and 1 (for instance $\Phi(-\infty)=0, \Phi(+\infty)=1$ ). The function $\Phi$ then necessarily fulfills the second order equation

$$
\left\{\begin{array}{l}
\Phi^{\prime \prime}(\xi)+c \Phi^{\prime}(\xi)+f(\Phi(\xi))=0, \quad-\infty<\xi<+\infty \\
0 \leq \Phi \leq 1, \quad \Phi(-\infty)=0, \Phi(+\infty)=1, \quad \Phi^{\prime}>0 .
\end{array}\right.
$$

The following result on existence and stability of traveling waves is well known.

Theorem 1.1 (see [8]) Let $f \in C^{1}[0,1]$ be satisfying the above conditions. Then there exists a unique (except for translations) monotone traveling front with range $[0,1]$, i.e., there exists a unique $c^{*}$ and a unique (except for translations) monotone solution $\Phi$ of (5).

Furthermore, suppose that $u_{0}$ is piecewise continuous, $0 \leq u_{0}(x) \leq 1$ for all $x \in \mathbb{R}$, and

$$
\liminf _{x \rightarrow+\infty} u_{0}(x)>\alpha, \quad \limsup _{x \rightarrow-\infty} u_{0}(x)<\alpha .
$$

Then there exist $x_{0} \in \mathbb{R}, K, \omega>0$, such that the solution $u$ to (4) satisfies

$$
\left|u(x, t)-\Phi\left(x-c^{*} t-x_{0}\right)\right|<K e^{-\omega t}, \quad x \in \mathbb{R}, \quad t>0 .
$$

From the numerical point of view, one of the main difficulties in the approximation of the profile $\Phi$ of the traveling wave solutions and their propagation speed $c$ is the need to set a finite computational domain. While the solution $u$ evolves into the traveling wave, it also moves left or right at velocity $c$ and it eventually may leave the chosen finite computational domain. A natural approach is to perform the change of variables $u(x, t)=v(x-c t, t)$, so that the resulting initial value problem for $v$ is given by $v_{t}=v_{x x}+c v_{x}+f(v)$ in the whole real line, with $v(x, 0)=u_{0}(x)$. 
Then the solution $v$ converges to a standing wave of the type $\Phi_{a}$, defined as $\Phi_{a}(x)=\Phi(x-a)$, for some $a \in \mathbb{R}$, which leads to the profile of the traveling wave. However, in general, the value of $c$ is not known a priori.

In this Note we introduce a nonlocal change of variables leading to a new nonlocal Cauchy problem, a variant of the reaction-diffusion above, that leads simultaneously to the traveling wave profile and speed.

\section{Preliminaries on the non local problem}

To overcome this difficulty, several approaches have been developed in the literature. One possibility, (see [4]) is to consider the speed of propagation $c$ as an appropriate function of the solution $u$ and incorporate this function in the $v$ equation. As a matter of fact, if we multiply by $\Phi$ in (5), integrate by parts and use the behavior of $\Phi$ at $x \rightarrow \pm \infty$, we get that

$$
c=-\frac{F(1)}{\left\|\Phi^{\prime}\right\|_{L^{2}(\mathbb{R})}^{2}}
$$

where $F(u)=\int_{0}^{u} f(s) d s$.

If we expect $v(\cdot, t)$ to converge, as $t \rightarrow+\infty$, to a translate of the traveling wave profile $\Phi$, we may also expect

$$
\lambda(v)=\frac{F(1)}{\left\|v_{x}\right\|_{L^{2}(\mathbb{R})}^{2}},
$$

to converge to $c$. This motivates the study of the nonlocal equation

$$
v_{t}=v_{x x}-\frac{F(1)}{\left\|v_{x}\right\|_{L^{2}(\mathbb{R})}^{2}} v_{x}+f(v), \quad x \in \mathbb{R}, t>0 ; \quad v(x, 0)=u_{0}(x) .
$$

With a different argument, using the so-called "phase condition", the authors in [4] obtained a partial differential algebraic equation, which is equivalent to the following nonlocal equation in the whole real line

$$
v_{t}=v_{x x}-\frac{\left\langle f(v), v_{x}\right\rangle_{L^{2}(\mathbb{R})}}{\left\|v_{x}\right\|_{L^{2}(\mathbb{R})}^{2}} v_{x}+f(v), \quad x \in \mathbb{R}, t>0 ; \quad v(x, 0)=u_{0}(x) .
$$

In [4] the authors implement appropriate numerical schemes to (10) to approximate the traveling wave solution and in [5] they analyze the stability properties of the traveling wave of (10). But they do not analyze the relation between (10) and its truncation to a finite interval neither the stability properties of the equilibria in a finite interval. Related non local equations have been treated in [11] where the Evans function for non local equations has been developed to analyze the spectrum.

Our first main result, concerning the asymptotic convergence towards the traveling wave profile and speed for this non-local problem, is as follows:

Theorem 2.1 Under the hypotheses of Theorem 1.1 and assuming further that $u_{0}^{\prime} \in L^{1}(\mathbb{R}) \cap L^{2}(\mathbb{R})$, the nonlocal problem (9) is well-posed and its solution $v$ is given by

$$
v(x, t):=u\left(x+\gamma_{u}(t), t\right), \quad x \in \mathbb{R}, \quad t>0,
$$

with

$$
\gamma_{u}(t):=-\int_{0}^{t} \frac{F(1)}{\left\|u_{x}(\cdot, s)\right\|_{L^{2}(\mathbb{R})}^{2}} d s, \quad t>0,
$$

where $u$ denotes the unique classical solution to (4). In particular, for each $x^{*} \in \mathbb{R}$, the function $\Phi\left(\cdot-x^{*}\right)$ is a "stationary" solution of (9) (standing wave).

Let $\omega_{0}=\min \{\beta, \gamma\}>0$, with $\beta=\min \left\{-f^{\prime}(0),-f^{\prime}(1)\right\}>0$ and $-\gamma<0$ the largest real part of any non zero eigenvalue of the operator

$$
L_{0}^{\infty} w:=w^{\prime \prime}+c^{*} w^{\prime}+f^{\prime}(\Phi) w, \quad \infty<x<\infty,
$$

see [10]. Then for any $\bar{\omega}<\omega_{0}$ there exist $x^{*} \in \mathbb{R}$ and a positive constant $C$ such that

$$
\sup _{x \in \mathbb{R}}\left|v(x, t)-\Phi\left(x-x^{*}\right)\right|+\left|\lambda_{v}(t)-c^{*}\right| \leq C_{1} e^{-\bar{\omega} t}, \quad t>0,
$$

where $c^{*}$ is the propagation speed in Theorem 1.1, $\lambda_{v}$ as in (8) and $\Phi$ the unique (except for translations) solution to (5)).

This theorem can be proved using the well known results about the local problem (4) (see Theorem 1.1 above) and analyzing carefully the change of variables that transforms it into the modified nonlocal one (9). 


\section{Stationary solutions for a truncated nonlocal problem}

This Theorem 2.1 is relevant from a numerical analysis and computational viewpoint. Indeed, it guarantees that, if we fix a sufficiently large computational domain, the core of the solution will not leave this window. Thus, in view of the asymptotic convergence result as $t$ tends to infinity, a suitable approximation of this nonlocal problem should enable us to approximate the traveling wave profile. But, when implementing a numerical scheme on a truncated bounded domain, we will need also to impose some "artificial" boundary conditions. We choose non-homogeneus Dirichlet boundary conditions, which emulate the behavior of the profile at $\pm \infty$ on the boundaries of the domain.

Summarizing, in practice, we will need to consider the following dynamics generated by the nonlocal reactiondiffusion problem in the bounded interval $(a, b)$ :

$$
\left\{\begin{array}{l}
v_{t}=v_{x x}-\frac{F(1)}{\left\|v_{x}(\cdot)\right\|_{L^{2}(a, b)}^{2}} v_{x}+f(v), \quad x \in(a, b), \quad t>0 \\
v(a, t)=0 ; v(b, t)=1, \quad t>0 \\
v(x, 0)=u_{0}(x), \quad x \in(a, b) .
\end{array}\right.
$$

The well posedness of this system in the space $H=\left\{u \in H^{1}(a, b), u(a)=0, u(b)=1\right\}$ follows standard arguments (notice that if $u \in H$ then $\left\|u_{x}\right\|_{L^{2}(a, b)}^{2} \geq(b-a)^{-1}$ and therefore the nonlocal term is not singular).

Our second main result refers to the asymptotic behavior of the solutions of this new problem. In particular, we are interested on its stationary solutions, its stability properties and its relations with the profile $\Phi$ of the traveling wave of the original problem.

It is easy to see that the stationary solutions of (15) depend only on the length of the interval $(a, b)$ and not on the actual value of its extremes.

Using appropriate phase plane techniques, we prove the following:

Theorem 3.1 For a fixed interval $(a, b)$ we denote $r=b-a$. Then,

(i) (Existence and uniqueness) There exists one and only one stationary solution $\Phi_{(a, b)}$ of $(15)$ with $0 \leq \Phi_{(a, b)}(x) \leq 1$. Moreover, this solution is strictly monotone increasing.

(ii) (Approximation of the speed) Let $\lambda_{(a, b)}=-F(1) /\left\|\Phi_{(a, b)}^{\prime}\right\|_{L^{2}(a, b)}^{2}$. Then, $\lambda_{(a, b)}=\lambda_{(\hat{a}, \hat{b})}$ if $b-a=\hat{b}-\hat{a}$ and $\lambda_{(a, b)} \rightarrow c^{*}$ as $b-a \rightarrow+\infty$, where $c^{*}$ is the speed of propagation of the traveling wave of the original problem (4).

(iii) (Approximation of the profile) Let us denote by $\theta$ the unique point in $(a, b)$ which satisfies $\Phi_{(a, b)}(\theta)=1 / 2$ and let us denote by $\Phi_{r}(x)=\Phi_{(a, b)}(x+\theta)$ for $x \in(a-\theta, b-\theta)$. Let also $a(r)=a-\theta$ and $b(r)=b-\theta$ (which truly depend only on $r)$. Let us normalize in a similar way the traveling wave solution, so that $\Phi_{\infty}(0)=1 / 2$. Then

$$
\left\|\Phi_{r}-\Phi_{\infty}\right\|_{W^{1, \infty}(\mathbb{R})}+\left\|\Phi_{r}^{\prime}-\Phi_{\infty}^{\prime}\right\|_{L^{2}(\mathbb{R})} \rightarrow 0, \quad \text { as } r \rightarrow \infty,
$$

where the function $\left\{\Phi_{r}\right\}$ is extended by 0 to the left of $a(r)$ and by 1 to the right of $b(r)$.

\section{Asymptotic stability of the stationary solutions of the nonlocal problem}

Our last main result ensures the stability of the stationary solutions of the nonlocal problem. This result is particularly relevant from a numerical analysis and computational viewpoint since it guarantees that when solving accurately the local problem for a sufficiently large time one will get an accurate approximation of the traveling wave profile and its speed.

The equilibrium $\Phi_{r}$ will be asymptotically stable if the spectrum of the linear operator $L^{r}: D\left(L^{r}\right) \subset L^{2}(a, b) \rightarrow$ $L^{2}(a, b)$ with $D\left(L^{r}\right)=H^{2}(a, b) \cap H_{0}^{1}(a, b)$, given by

$$
L^{r} w:=w_{x x}+\lambda\left(\Phi_{r}\right) w_{x}+f^{\prime}\left(\Phi_{r}\right) w+\frac{2 \lambda\left(\Phi_{r}\right)}{\left\|\Phi_{r}^{\prime}\right\|_{2}^{2}} \Phi_{r}^{\prime} \int w \Phi_{r}^{\prime \prime}
$$

is contained in the left half of the complex plane.

Observe that $L^{r} w=L_{0}^{r} w+\Pi_{r}(w)$, where $L_{0}^{r} w=w_{x x}+\lambda\left(\Phi_{r}\right) w_{x}+f^{\prime}\left(\Phi_{r}\right) w$ is a "standard" Sturm-Liouville type operator and $\Pi_{r}(w)$ is the nonlocal linear operator, which has 1-dimensional rank. This operator is of the form $w \rightarrow A\langle w, B\rangle$ with $A(\cdot)=\frac{2 \lambda\left(\Phi_{r}\right)}{\left\|\Phi_{r}^{\prime}\right\|_{2}^{2}} \Phi_{r}^{\prime}(\cdot)$ and $B(\cdot)=\Phi_{r}^{\prime \prime}(\cdot)=-\lambda\left(\Phi_{r}\right) \Phi_{r}^{\prime}(\cdot)-f\left(\Phi_{r}(\cdot)\right)$ and it is a bounded operator from $L^{2}$ to $L^{2}$ with finite rank. Several properties of the operator $L^{r}$ are inherited from the operator $L_{0}^{r}$ : both operators have the same domain and compact resolvent and therefore their spectra is only discrete, formed by eigenvalues with finite multiplicity. Nevertheless, all the eigenvalues of operator $L_{0}^{r}$ are real (there is a standard change of variables 
transforming $L_{0}^{r}$ to a selfadjoint operator) but the operator $L^{r}$ may not have this property. Actually, unless $A \equiv B$ operator $L^{r}$ is not selfadjoint.

There are several results on the spectra of operators of the form $w \rightarrow L_{0}^{r}(w)+A\langle w, B\rangle$ but none of them guarantees that, for our particular case, the spectrum lies in the half complex plane with negative real part. Actually, with the known results in the literature we are not even able to ensure that the spectrum of $L$ is real (see $[6,7,9]$ and references therein, for results in this direction).

One important observation is that in the case that the interval is the complete real line, that is $r=\infty$, then $\Phi_{\infty}^{\prime}$ is the eigenfunction associated to the eigenvalue 0 for the operator $L_{0}^{\infty}$ and therefore the operator $L^{\infty}=L_{0}^{\infty}+\pi_{\infty}(w) \Phi_{\infty}^{\prime}$ has a special structure that will allow us to show that $\sigma\left(L^{\infty}\right)=\sigma\left(L_{0}^{\infty}\right)$ and that $0 \in \sigma\left(L^{\infty}\right)$ with multiplicity 1 . The fact that $\Phi_{r}^{\prime}$ is not an eigenfunction of $L_{0}^{r}$, for finite $r$ (as a matter of fact $\Phi_{r}^{\prime}$ does not even satisfy homogeneous Dirichlet boundary conditions) will not permit us to perform a similar argument in a bounded interval. Paradoxically, the analysis in the whole real line is "simpler" than the analysis in a bounded interval.

Nevertheless we will be able to prove the asymptotic stability of the stationary solution of the non local problem (15) for large enough intervals using a perturbative method. Let us highlight the main steps of the proof of stability. We refer to [2] for the details of the proofs.

\section{Step 1.- On the spectrum of $L^{r}$.}

From the convergence of $\Phi_{r}$ and $\lambda\left(\Phi_{r}\right)$ obtained above in Theorem 3.1 and using the structure of the operator $L^{r}$, we easily show the following result:

Proposition 4.1 There exist $\rho_{0} \in \mathbb{R}^{+}$and $\phi \in(\pi, 2 \pi)$ such that if we define the sector $\Sigma_{\rho_{0}, \phi}=\{z \in \mathbb{C}, \mid \arg (z-$ $\left.\left.\rho_{0}\right) \mid>\phi\right\}$, then $\sigma\left(L^{r}\right) \subset \Sigma_{\rho_{0}, \phi}$ for all $r \geq 1$.

Moreover, we are also able to show:

Proposition 4.2 There is no real eigenvalue $\mu \geq 0$ in $\sigma\left(L^{r}\right)$.

Step 2.- On the spectrum of $L^{\infty}$.

This operator is the one associated to the linearization around the stationary state $\Phi_{\infty}$, which can be written as $L^{\infty}=L_{0}^{\infty}+\Pi_{\infty}$, where $L_{0}^{\infty}$ is given by $(13)$ and $\Pi_{\infty}$ stands for the linear non local operator

$$
\Pi_{\infty}(w)=\frac{-2 \lambda\left(\Phi_{\infty}\right)}{\left\|\Phi_{\infty}^{\prime}\right\|_{2}^{2}}\left\langle\Phi_{\infty}^{\prime}, w_{x}\right\rangle \Phi_{\infty}^{\prime} .
$$

The fact that both $L_{0}^{\infty}$ and $L^{\infty}$ are sectorial operators and that $\Phi_{\infty}^{\prime}$ is the eigenfunction of $L_{0}^{\infty}$ associated to the eigenvalue $\mu=0$, allows us to prove

Proposition $4.3 \sigma\left(L^{\infty}\right)=\sigma\left(L_{0}^{\infty}\right)$ and $0 \in \sigma\left(L^{\infty}\right)$. In particular

(i) The essential spectrum $\sigma_{\text {ess }}\left(L^{\infty}\right) \subset\left\{z \in \mathbb{C}: \operatorname{Re} z \leq \max \left\{f^{\prime}(0), f^{\prime}(1)\right\}\right\}$.

(ii) There exists $\left.0<\nu<-\max \left\{f^{\prime}(0), f^{\prime}(1)\right\}\right\}$ such that $\sigma\left(L^{\infty}\right) \cap\{z \in \mathbb{C}: \operatorname{Re} z \geq-\nu\}=\{0\}$. Moreover, the eigenfunction associated to the eigenvalue $\mu=0$ is $\Phi_{\infty}^{\prime}$ and it is an algebraically simple eigenvalue of $L^{\infty}$, that is, $\operatorname{Ker}\left(\left(L^{\infty}\right)^{2}\right)=\operatorname{Ker}\left(L^{\infty}\right)=\operatorname{span}\left\{\Phi_{\infty}^{\prime}\right\}$.

Step 3.- Passing to the limit.

The asymptotic stability of the stationary solution $\Phi_{r}$ of the non local problem for large enough $r$ is obtained via the convergence of the spectrum of the operator $L^{r}$ to $L^{\infty}$ in bounded sets of the complex plane away from the essential spectrum of $L^{\infty}$. In order to prove this spectral convergence we will use the theory of regular convergence developed in [12]. Notice that in [3] they show this convergence for the local operators only. The use of the theory of exponential dichotomies becomes an essential ingredient in the proof. Adapting the proof of [3] to our operators, which have a non local term but which is compact, will allow us to prove the required spectral convergence. Putting together this spectral convergence and Proposition 4.2, we are able to show:

Theorem 4.1 For every fixed $\varepsilon>0$, there exists an $r_{0}>0$ such that for all $r \geq r_{0}$ we have $\sigma\left(L_{r}\right) \cap\{\operatorname{Re} z>$ $-\nu+\varepsilon\}=\{s(r)\}$. Moreover, $s(r)<0$ is a simple eigenvalue of $L_{r}$ and $s(r) \rightarrow 0$ as $r \rightarrow+\infty$. In particular, the unique stationary solution of $(15)$ is asymptotically stable.

\section{Numerical examples}

In this section we propose numerical examples showing the efficiency of the methods analyzed in this paper. We consider the prototypical Nagumo equation, given by (4) with $f(u)=u(1-u)(u-\alpha), \alpha \in(0,1 / 2)$. In this case an explicit travelling wave solution $u(x, t)=\Phi(x-c t)$ is known, where $\Phi(x)=\left(1+e^{-x / \sqrt{2}}\right)^{-1}$ and $c=\sqrt{2}(\alpha-1 / 2)$.

We fix $J>0, \alpha=1 / 4$, a final time $T=150$ and consider the numerical integration of (15) in the interval $[-J, J]$ with $[0,1]$-Dirichlet boundary conditions. We apply the method of lines and use for the spatial discretization standard finite differences formulas, centered for the approximation of $v_{x}$, on the uniform grid $x_{j}=-J+j \Delta x$, 

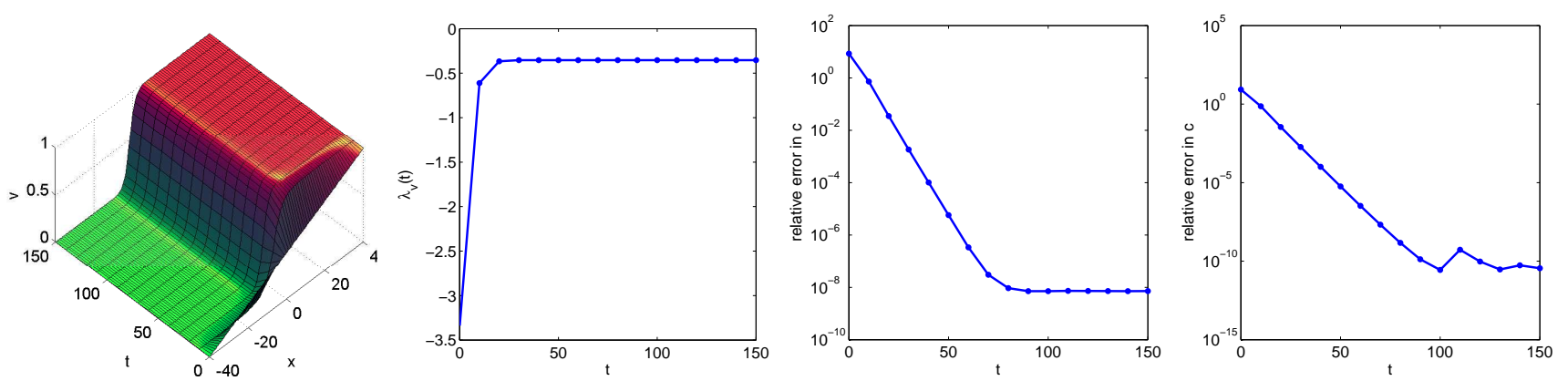

Figure 1. Solution for $u_{0}(x)=\frac{x+J}{2 J}$, evolution of $\lambda_{v}$ and evolution of the error in the approximation of $c$ for $J=40$ and $\Delta x=0.1$ and 0.025 .
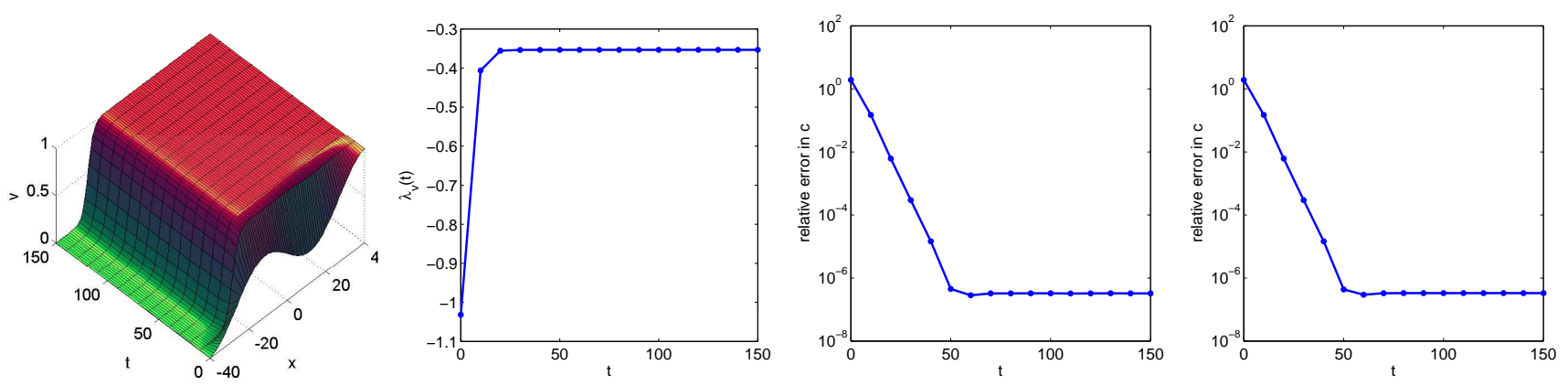

Figure 2. Solution for $u_{0}(x)=\frac{1}{2}\left(1+0.53 \frac{x}{J}+0.47 \sin \left(-\frac{3 \pi x}{2 J}\right)\right)$, evolution of $\lambda_{v}$ and evolution of the error in the approximation of $c$ for $J=40$ and $\Delta x=0.1$ and 0.025 .

$1 \leq j \leq M-1, \Delta x=2 J / M$. We consider different values of $J$ and $M$ and two different initial data. The nonlocal term $\lambda_{v}$ is approximated by using the scalar product of the vector with the values of $v_{x}$ at the grid points $x_{j}$. Our results are shown in Figures 1 and 2.

Acknoweledgements. The first author was partially supported by Grants MTM2009-07540 and MTM2006-08262 MICINN, Grant GR58/08 UCM-BSCH Grupo 920894 and PHB2006-003 PC, MICINN, Spain. The second author was partially supported by Grants MTM 2008-03541 and MTM 2010-19510, MICINN, Spain. The third author was partially supported by Grant MTM2008-03541, MICINN, Spain, ERC Advanced Grant FP7-246775 NUMERIWAVES, ESF Research Networking Programme OPTPDE and Grant PI2010-04 of the Basque Government.

\section{References}

[1] D. G. Aronson, H. F. Weinberger, Multidimensional nonlinear diffusion arising in population genetics, Adv. Math. 30 (1978) $33-76$.

[2] J. M. Arrieta, M. López-Fernández, E. Zuazua, Approximating traveling waves by equilibria of nonlocal equations, Preprint, 2011.

[3] W. J. Beyn, J. Rottman-Matthes, Resolvent estimates for boundary value problems on large intervals via the theory of discrete approximations, Numer. Funct. Anal. Optim. 28 (2007) 603-629.

[4] W. J. Beyn, V. Thümmler, Freezing solutions of equivariant evolution equations, SIAM J. Appl. Dyn. Syst. 3 (2004) 85-116.

[5] W. J. Beyn, V. Thümmler, Phase conditions, symmetries, and PDE continuation, Numerical continuation methods for dynamical systems, pp. 301-330, Underst. Complex Syst., Springer, Dordrecht, 2007.

[6] F.A. Davidson, N. Dodds, Spectral properties of non-local uniformly-elliptic operators, Electron. J. Differential Equations, 126 (2006), $15 \mathrm{pp}$.

[7] N. Dodds, Further spectral properties of uniformly elliptic operators that include a non-local term, Appl. Math. Comput., 197 (2008), pp. 317-327.

[8] P. C. Fife, J. B. McLeod, The approach of solutions of nonlinear diffusion equations to traveling front solutions, Arch. Rational Mech. Anal. 65 (1977) 335-361.

[9] P. Freitas, Non-local reaction diffusion equations, Fields Institute Communications, 21 (1999), pp. 187-204.

[10] D. Henry, Geometric theory of semilinear parabolic equations, Lecture Notes in Mathematics 840, Springer, Berlin, 1981.

[11] T. Kapitula, N. Kutz, B. Sandstede, The Evans function forn Nonlocal Equations, Indiana Univ. Math. J. 53 (2004), no. 4, 10951126.

[12] G. Vainikko, Funktionalanalysis der Diskretisierungsmethoden, Leipzig 1976. 